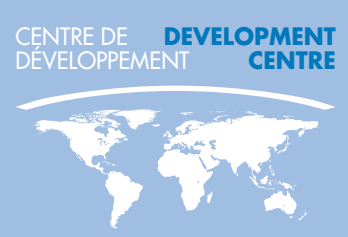

\title{
L'éducation au service de la mobilité sociale ascendante en Amérique latine
}

\author{
par Christian Daude
}

- Les revenus sont très inégalement répartis en Amérique latine, de même que les possibilités de mobilité sociale ascendante.

- Le développement de la petite enfance est un puissant mécanisme égalisateur du champs social.

- Une éducation secondaire plus généralisée et de meilleure qualité est un élément clé. Les réformes devront s'appuyer sur une meilleure administration des écoles qui associe flexibilité et transparence à un système moderne d'évaluation et d'incitations pour les gestionnaires d'établissements scolaires et les enseignants.

La constance des niveaux éducatifs atteints d'une génération à l'autre (les enfants et les parents ayant le même niveau d'instruction) constitue un facteur clé de la persistance des écarts de rémunération entre les différentes couches de la société. Parallèlement, le renforcement du capital humain - qui résulte de politiques éducatives de qualité - est un vecteur déterminant de la croissance économique, à la fois à travers son impact positif direct sur la productivité du travail et ses complémentarités avec l'innovation et l'absorption de nouveaux savoirs dans le processus de production.

\section{L'inégalité des chances reste très marquée en Amérique latine}

Alors qu'elle détient le record mondial de l'inégalité des revenus, I'Amérique latine reste aussi très inégalitaire dans les chances d'ascension sociale. L'accès aux services éducatifs, aussi bien en termes quantitatifs que qualitatifs, est faible pour les couches moyennes de la région comparé à leurs homologues des pays de I'OCDE mais aussi aux ménages latino-américains aisés. C'est pourquoi les politiques publiques visant à atténuer les inégalités entre générations et au sein d'une même génération se justifient amplement. Pour que les politiques éducatives favorisent la mobilité ascendante, il faut qu'elles intègrent - dès la phase d'élaboration des considérations d'équité.

\section{Probabilité d'atteindre un niveau d'instruction plus élevé que celui de ses parents en Amérique latine}

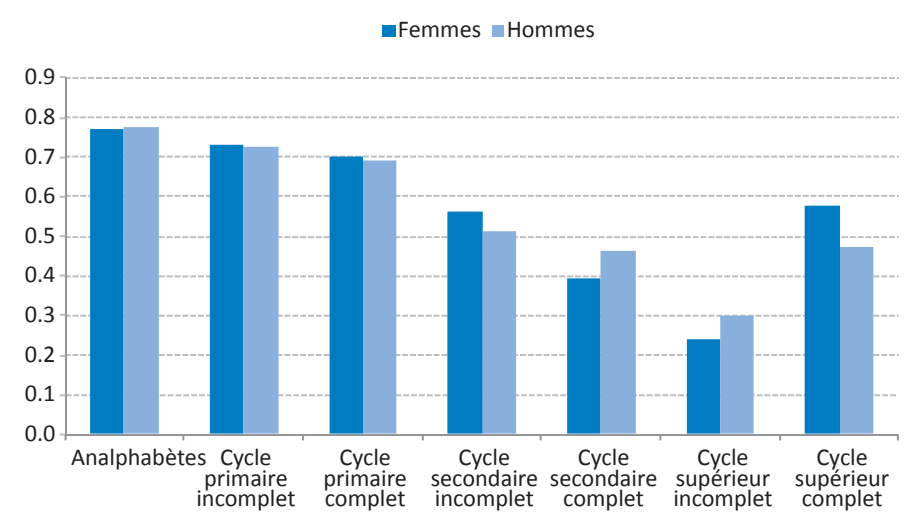

Note : Les barres représentent la probabilité moyenne estimée, pour un enfant, d'atteindre un niveau d'instruction plus élevé que ses parents, sauf pour la catégorie « cycle supérieur complet », où elles représentent la probabilité d'atteindre le même niveau d'instruction. Les enfants de l'échantillon sont des hommes et des femmes âgés de 25 à 44 ans au moment de l'enquête.

Source : D'après les enquêtes Latinobarómetro réalisées dans 18 pays d'Amérique latine en 2008.

Point positif : une mobilité ascendante semble s'opérer à travers l'éducation pour ceux dont les antécédents familiaux sont les plus défavorables - et la mobilité descendante est très improbable pour ceux au sommet de l'échelle. Néanmoins, les couches moyennes semblent bloquées, le niveau d'instruction des enfants issus de ces milieux atteignant un pic autour de l'achèvement d'une scolarité secondaire. 
L'écart avec les individus dont les parents ont fait des études supérieures reste substantiel. Ainsi, sur 100 enfants dont les parents ont effectué une scolarité incomplète dans le secondaire, une dizaine termine ses études supérieures, contre 58 femmes et 47 hommes lorsque les parents ont achevé des études supérieures. Il apparaît donc qu'environ $80 \%$ des Latino-américains de 25-44 ans ont des parents qui ne sont pas allés au terme du secondaire ou se sont arrêtés avant.

\section{Quelles politiques pour améliorer la mobilité sociale en Amérique latine?}

- Le développement de la petite enfance accroît singulièrement les opportunités pour les populations défavorisées des pays en développement. Il est une condition préalable de l'égalité des chances plus tard dans la vie. Une hausse des dépenses publiques au profit de l'éducation préscolaire et une augmentation des taux de scolarisation atténuent la relation entre faible niveau d'instruction des parents et résultats scolaires des enfants dans le secondaire.

- L'universalisation de l'enseignement secondaire pour les enfants des couches défavorisées et moyennes de la région est cruciale pour rompre la transmission des inégalités. Actuellement, la scolarité obligatoire ne couvre que neuf années d'éducation. Il est possible de la porter à 12 années. Cet allongement devrait avoir un impact maximal pour les couches moyennes. Des incitations matérielles supplémentaires pourraient se révéler nécessaires pour les ménages plus pauvres, afin qu'ils respectent cette obligation.

- Le relèvement qualitatif de l'éducation - un objectif essentiel par nature - accroîtrait aussi l'équité. Il réduirait le fossé entre enseignement public et enseignement privé en lissant les écarts dans l'acquisition de compétences entre les couches défavorisées et moyennes, d'une part, et les couches aisées, d'autre part. Il endiguerait également les abandons et susciterait une demande accrue d'éducation, étant donné les rendements plus élevés attendus de l'investissement éducatif. Grâce à de réelles perspectives d'amélioration du niveau d'instruction, les parents des couches moyennes pourraient accueillir favorablement ces mesures, surtout dans l'enseignement secondaire. Comment améliorer la qualité de l'éducation ? L'optimisation de l'administration scolaire, une flexibilité plus grande conjuguée à une transparence accrue, un système moderne d'évaluation et d'incitations pour les gestionnaires des établissements scolaires sont autant d'éléments susceptibles d'accroître le rendement des dépenses courantes. De même, les réformes doivent prévoir des régimes d'incitations efficaces pour les enseignants ainsi qu'un renforcement continu de leurs compétences et de leurs qualifications.

\section{Pour approfondir la question :}

D'Addio, A.-C. (2007), "Intergenerational Transmission of Disadvantage: Mobility or Immobility across Generations? A review of the Evidence in OECD Countries," Documents de travail de l'OCDE sur les affaires sociales, l'emploi et les migrations, $\mathrm{n}^{\circ} 52, \mathrm{OCDE}$, Paris.

Daude, C. (2010), "Ascendance by descendants? On social mobility in Latin America", Document de travail, Centre de développement de I'OCDE, Paris, à paraître.

OCDE (2008), « Mobilité intergénérationnelle - quel est son effet sur I'inégalité des revenus ? », Croissance et inégalités : distribution des revenus et pauvreté dans les pays de I'OCDE, OCDE, Paris, pp. 203-221.

OCDE (2010a), Perspectives économiques de l'Amérique latine 2011 : I'Amérique latine, une région de classes moyennes?, Centre de développement de I'OCDE, Paris.

OCDE (2010b), « Une affaire de famille : la mobilité sociale intergénérationnelle dans les pays de I'OCDE », Réformes économiques : Objectif croissance 2010, OCDE, Paris, pp. 191207.
Les lecteurs sont invités à citer ou reproduire les informations des Repères du Centre de développement de I'OCDE dans leurs propres publications. En échange, le Centre demande les remerciements de rigueur ainsi qu'un exemplaire de la publication. Le texte intégral des Repères et d'autres informations sur le Centre de développement et ses travaux sont disponibles sur : www.oecd.org/dev
Centre de développement de I'OCDE

2 , rue André-Pascal,

75775 Paris Cedex 16, France

Tél : $33(0) 145.24 .82 .00$

Fax : 33 (0) 144.30 .61 .49

mél : dev.contact@oecd.org 